\title{
Development of Sensor Surfaces Using Poly-( $N$-vinylformamide) for Sensitive Detection of 2,4,6-Trinitrotoluene by Displacement Method on a Surface Plasmon Resonance Sensor
}

\author{
Takeshi Onodera, ${ }^{1 *}$ Ryosuke Ishii, ${ }^{2}$ Rui Yatabe, ${ }^{1}$ and Kiyoshi Toko ${ }^{1,2}$ \\ ${ }^{1}$ Research and Development Center for Taste and Odor Sensing, Kyushu University, \\ 744 Motooka, Nishi-ku, Fukuoka-shi, Fukuoka 819-0395, Japan \\ ${ }^{2}$ Graduate School of Information Science and Electrical Engineering, Kyushu University, \\ 744 Motooka, Nishi-ku, Fukuoka-shi, Fukuoka 819-0395, Japan \\ (Received October 8, 2015; accepted February 8, 2016)
}

Keywords: surface plasmon resonance, displacement, TNT, poly- $N$-vinylformamide, antigen-antibody interaction

To detect 2,4,6-trinitrotoluene (TNT) using a displacement method over a surface plasmon resonance immunosensor, we developed sensor surfaces modified with a poly-( $N$-vinylformamide$c o$-vinylamine), which was synthesized from a monomer, $N$-vinylformamide, and was hydrolyzed to produce amine groups, and TNT analogs. Poly-( $N$-vinylformamide-co-vinylamine) that reacted with succinic acid was used as a matrix of a sensor surface for increasing the number of antibody binding sites. 2,4-Dinitrophenyl glycine, 3-nitrophenyl acetic acid, and 4-nitrophenyl acetic acid were immobilized on the polymer-modified sensor surfaces. We evaluated the suppression ability of the sensor surface for nonspecific adsorption and determined the limit of detection (LOD) for TNT. As a result, a sensor chip suppressed nonspecific adsorption for $1 \mathrm{mg} / \mathrm{mL}$ bovine serum albumin and lysozyme. TNT was measured by the displacement method, and the LOD achieved over a 3-nitrophenyl acetic acid-modified sensor chip was $0.9 \mathrm{ng} / \mathrm{mL}$ (ppb).

\section{Introduction}

Security checks at airports have been tight since the terrorist attacks in the USA on 11 September 2001. However, terrorist attacks using bombs sometimes occur in urban areas. An improvised explosive device (IED), which is fabricated with commonly available materials, can be set in a residential area in a troubled region. To find and defuse bombs safely in urban and residential areas, a portable explosive detector with high sensitivity and selectivity is desired. For this purpose, we have developed an electronic dog nose based on a surface plasmon resonance (SPR) immunosensor. ${ }^{(1,2)}$ Displacement immunoassay is one of the rapid and sensitive detection methods for SPR measurement for 2,4,6-trinitrotoluene (TNT). ${ }^{(3)}$ Various types of sensor surfaces need to be prepared for any situation.

"Corresponding author: e-mail: onodera@ed.kyushu-u.ac.jp 
We have reported a sensor chip modified with an ethylene glycol-unit-containing self-assembled monolayer (SAM) and a sensor surface modified with polymer brush grown by surface initiated atom transfer radical polymerization (SI-ATRP) for displacement immunoassay. ${ }^{(3,4)}$ In the former study, we selected a suitable TNT analog among three types of dinitroaromatic compounds for highly sensitive detection. In the latter study, we developed a method to control the density of immobilized TNT analogs on the polymer brush to obtain the lowest limit of detection (LOD). We have also reported that a sensor chip modified with a synthesized polymer, poly-( $N$-vinylformamide) (poly-NVF), which has a controlled hydrolysis rate, resulted in poly-( $N$-vinylformamide-covinylamine) [poly-(NVF-co-VAm)] for an indirect competitive method.(5) The many amine groups in the polymer provide a large number of antibody binding sites to immobilize TNT analogs.

In this study, we tried to use mononitroaromatic compounds as TNT analogs for the binding site of a commercially available anti-TNT monoclonal antibody (anti-TNT Ab). Mononitroaromatic compounds may interact with the anti-TNT Ab weakly. It is expected that the dissociation of an antibody bound with a TNT analog on the sensor surface by TNT will be accelerated. ${ }^{(6)}$ We evaluated the suppression ability of the nonspecific adsorption of poly-(NVF-co-VAm) reacted with succinic anhydride. We fabricated sensor chips modified with the polymer and TNT analogs for the detection of TNT by displacement assay.

\section{Materials and Methods}

\subsection{Reagents and chemicals}

The following chemicals were used without further purification: a mouse anti-TNT monoclonal antibody (anti-TNT Ab) was purchased from Strategic Biosolutions (Ramona, CA, USA). A TNT solution (20.2 ppm) in MilliQ water was obtained from Chugoku Kayaku Co., Ltd. (Hiroshima, Japan). $\quad N$-(2,4-dinitrophenyl glycine)(DNP-gly), 4-nitrophenyl acetic acid (4-NP-aa), and 3-nitrophenyl acetic acid (3-NP-aa) were purchased from Tokyo Chemical Industry (Tokyo, Japan). Figure 1 shows these nitro aromatic compounds. $N$-vinylformamide (NVF) was obtained from Aldrich (St. Louis, MO, USA). VA-044 \{2,2'-Azobis[2-(2-imidazolin-2-yl)propane] dihydrochloride\}, which is an initiator of radical polymerization, was purchased from Wako Pure<smiles>Cc1c([N+](=O)[O-])cc([N+](=O)[O-])cc1[N+](=O)[O-]</smiles>

(a)<smiles>O=C(O)CNc1ccc([N+](=O)[O-])cc1[N+](=O)[O-]</smiles>

(b)

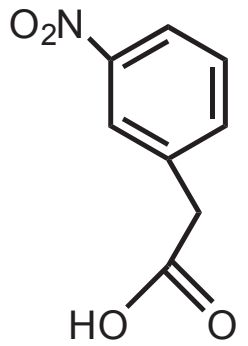

(c)

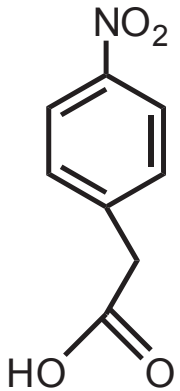

(d)

Fig. 1. Structural formulas of (a) TNT, (b) DNP-gly, (c) 3-NP-aa, and (d) 4-NP-aa. 
Chemical Industries (Osaka, Japan). Succinic anhydride was obtained from Nacalai Tesque (Kyoto, Japan). PEG6-COOH aromatic dialkanethiol was purchased from Senso Path Technologies (Bozeman, MT, USA). $N$-ethyl- $N$ '-(3-dimethylaminopropyl) carbodiimide (EDC) and $N$-hydroxysuccinimide (NHS) in an amine coupling kit (GE Healthcare Piscataway, NJ, USA) were used. All other chemicals were purchased either from Tokyo Chemical Industry, Japan, Wako Pure Chemical Industries, Inc., or Kanto Chemical, Co., Japan. All aqueous solutions were prepared using MilliQ deionized water obtained from a MilliQ-system (Millipore, Bedford, MA, USA).

\subsection{Polymer synthesis}

Poly-NVF can be polymerized from $N$-vinylformamide with the polymeric initiator VA044. ${ }^{(7)}$ Poly-NVF and poly-VAm were synthesized by the procedure described in Ref. 5 with slight modification. NVF and VA-044 were dissolved in MilliQ water, and each of the solutions was degassed for $1 \mathrm{~h}$. The solutions were mixed at a molar ratio of NVF:VA-044 $=4000: 1$. The polymerization was carried out for $24 \mathrm{~h}$ at $50^{\circ} \mathrm{C}$, and poly-NVF was obtained. Next, the poly-NVF was dissolved in $20 \%$ aqueous ethanol solution. When acetone was added, a resinlike polymer was precipitated. After that, the resinlike polymer was dissolved in water. After the polymer solution was dried with a vacuum freeze dryer (FDU-1200, Tokyo Rikakikai), a spongelike poly-NVF was obtained. Next, the poly-NVF was dissolved in $2 \mathrm{M} \mathrm{NaOH}$ solution at $20 \mathrm{mg} / \mathrm{mL}$. The hydrolysis of poly-NVF was carried out for $10 \mathrm{~min}$ or $4 \mathrm{~h}$ at $80^{\circ} \mathrm{C}$, and poly-(NVF-co-VAm) and poly-VAm solutions were obtained. After that, the solutions were neutralized and purified using dialysis tubes (Spectra/Por membrane, MWCO:3500, Spectrum) in MilliQ water. Finally, spongelike polymers were obtained after lyophilization using the vacuum freeze dryer.

Poly-(NVF-co-VAm) dissolved in $0.6 \mathrm{M}$ aqueous succinic anhydride solution at a concentration of $5 \mathrm{mg} / \mathrm{mL}$ was reacted for $24 \mathrm{~h}$ at $40{ }^{\circ} \mathrm{C}$. After that, the solution was neutralized and purified using a dialysis tube in MilliQ water at $4{ }^{\circ} \mathrm{C}$. Finally, a spongelike poly-(NVF-co-VAm) with succinic acid attached was obtained after lyophilization using the vacuum freeze dryer.

\subsection{Fabrication of sensor surface}

A bare Au sensor chip in a SIA Au kit (GE Healthcare) was used for sensor chip fabrication. Sensor chips were fabricated using a published procedure. ${ }^{(5)}$ The procedure is shown in Fig. 2. After the cleaning of the sensor chips, SAMs were formed on the sensor chips using PEG6-COOH aromatic dialkanethiol. To remove unreacted reagent from the sensor chip, the sensor chip was sonicated in ethanol with an ultrasonic cleaner. To activate the carboxyl terminal group of the SAM, a mixture of 0.4 M EDC and 0.1 M NHS solutions at a 1:1 ratio was dropped on the sensor chip surface and reacted for $1 \mathrm{~h}$. After rinsing with MilliQ water, a $50 \mathrm{mg} / \mathrm{mL}$ solution of poly(NVF-co-VAm) with succinic acid attached was reacted with the chip surface for $1 \mathrm{~h}$ and then the chip was rinsed with MilliQ water. Ethanol amine was dropped on the sensor chip to block the residual NHS ester. To immobilize the TNT analogs (DNP-gly, 3-NP-aa, and 4-NP-aa) by the amine coupling reaction, the carboxyl group of each analog was activated with EDC and NHS solutions. Then, the activated analogs were reacted with the amine groups of the polymer on the sensor chips. 


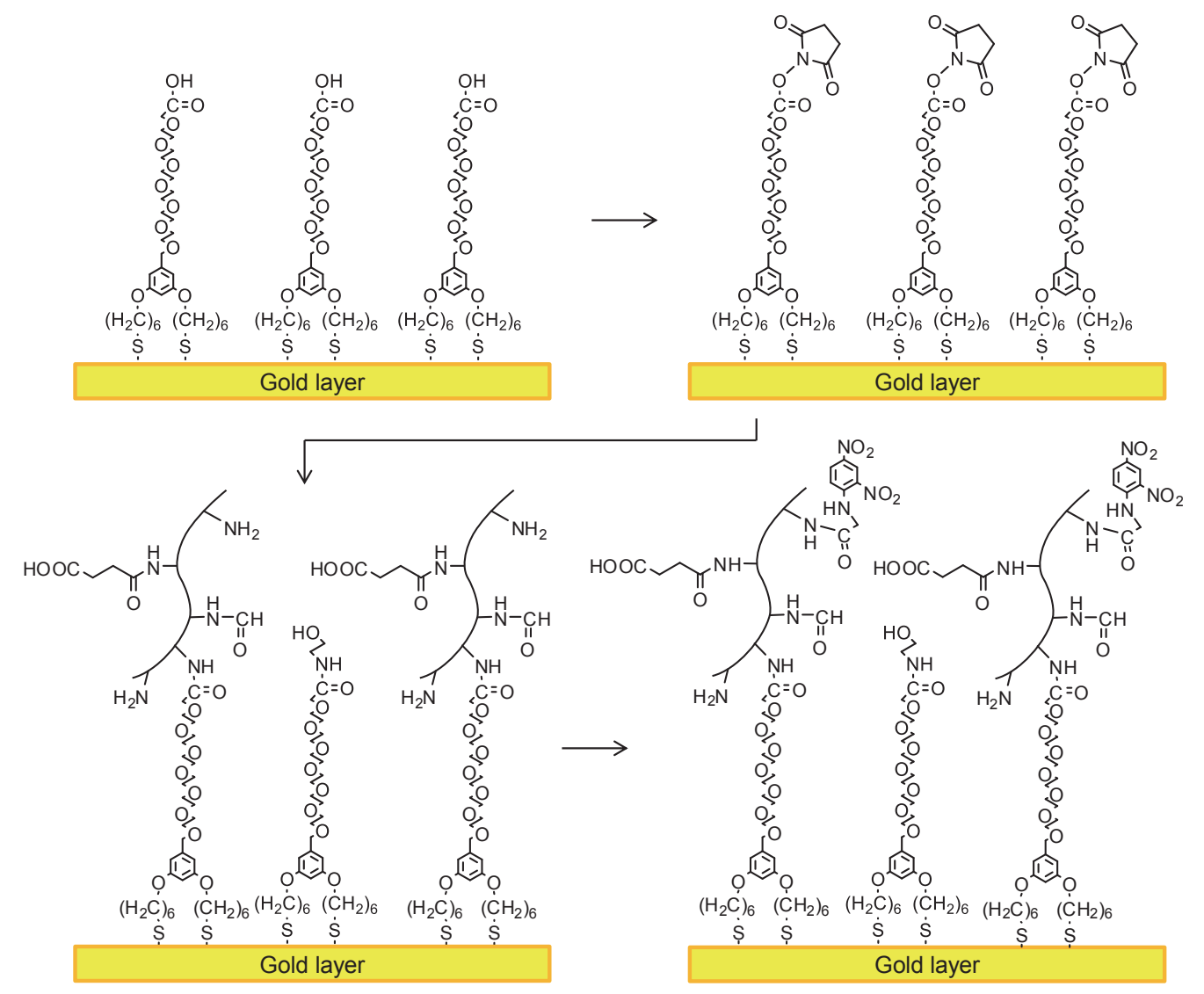

Fig. 2. (Color online) Fabrication of a sensor chip modified with poly-(NVF-co-VAm) reacted with succinic acid.

\subsection{Instrumentation and conditions}

Frontier Gold FTIR (PerkinElmer, MA, USA) with a universal ATR (1 reflection, diamond/ KRS5) was used to analyze the polymers by Fourier transform infrared spectroscopy.

The interaction between the TNT analog immobilized on the sensor surface and the anti-TNT $\mathrm{Ab}$ was analyzed with a BiacoreJ system (GE Healthcare). All measurements were performed at 25 ${ }^{\circ} \mathrm{C}$. PBST [ $50 \mathrm{mM}$ phosphate-buffered saline, $150 \mathrm{mM} \mathrm{NaCl}, 0.05 \%(\mathrm{v} / \mathrm{v})$ Tween 20, $\mathrm{pH}$ 7.2] was used as the running buffer at a flow rate of $14 \mu \mathrm{l} / \mathrm{min}$ (set at a low flow rate). TNT solutions ([20.2 $\mu \mathrm{g} / \mathrm{mL}(\mathrm{ppm})]$ ) of various concentrations were made by dilution with PBST.

\subsection{Evaluation of nonspecific adsorption of fabricated sensor chip}

A $25 \mu \mathrm{g} / \mathrm{mL}(\mathrm{ppm})$ anti-TNT Ab solution, a $1000 \mu \mathrm{g} / \mathrm{mL}$ BSA solution, and a $1000 \mu \mathrm{g} / \mathrm{mL}$ lysozyme solution were allowed to flow over the fabricated sensor chip for $2 \mathrm{~min}$. ${ }^{(8)}$ Regeneration of the sensor chip for anti-TNT Ab was carried out using $0.5 \%$ sodium dodecyl sulfate (SDS) for 2 min. SDS $(0.5 \%)$ or $3 \mathrm{M} \mathrm{NaCl}$ was used for the regeneration of BSA and lysozyme. 


\subsection{TNT detection using the displacement method}

In the displacement method, the antibody and the target solution do not need to be premixed before measurement as in the indirect competitive method. ${ }^{(9)}$ The procedure of the displacement method is described in Refs. 3 and 4. In this study, some parameters were changed. Figure 3 shows the procedure for the displacement immunoassay to detect TNT in this study. When $25 \mu \mathrm{g} / \mathrm{mL}$ (ppm) anti-TNT Ab was introduced to the TNT modified on the sensor surface, the increase in SPR response was due to the binding of anti-TNT Ab to the immobilized TNT analog. When the flow of anti-TNT Ab stopped, running buffer was automatically used, and the spontaneous dissociation of the bound antibody started immediately. Then, PBST without TNT or TNT in PBST was injected. The sensor response was defined at the injection point as $\Delta \theta_{\text {pre }}$. The response of PBST without TNT was used as a reference (control). When TNT at a high concentration was present in the buffer solution, dissociation from the analog was accelerated and the sensor response decreased markedly. Anti-TNT Ab bound to the TNT analog immobilized on the sensor surface was switched to bind to TNT instead of the TNT analog. The anti-TNT Ab-TNT complex that was formed broke away from the sensor surface. The sensor response after finishing the flow of PBST without TNT or TNT in PBST was defined as $\Delta \theta_{\text {post }}$. In this study, displacement ratio was defined using Eqs. (1) and (2).

$$
\begin{gathered}
\tau_{i}=\frac{\Delta \theta_{\mathrm{post}}}{\Delta \theta_{\mathrm{pre}}}(i=1,2, \ldots) \\
\text { Displacement ratio }=\frac{\tau_{0}-\tau_{i}}{\tau_{0}} \times 100(\%)
\end{gathered}
$$

Here, $\Delta \tau_{0}$ is the amount of remaining antibody when PBST without TNT was introduced.

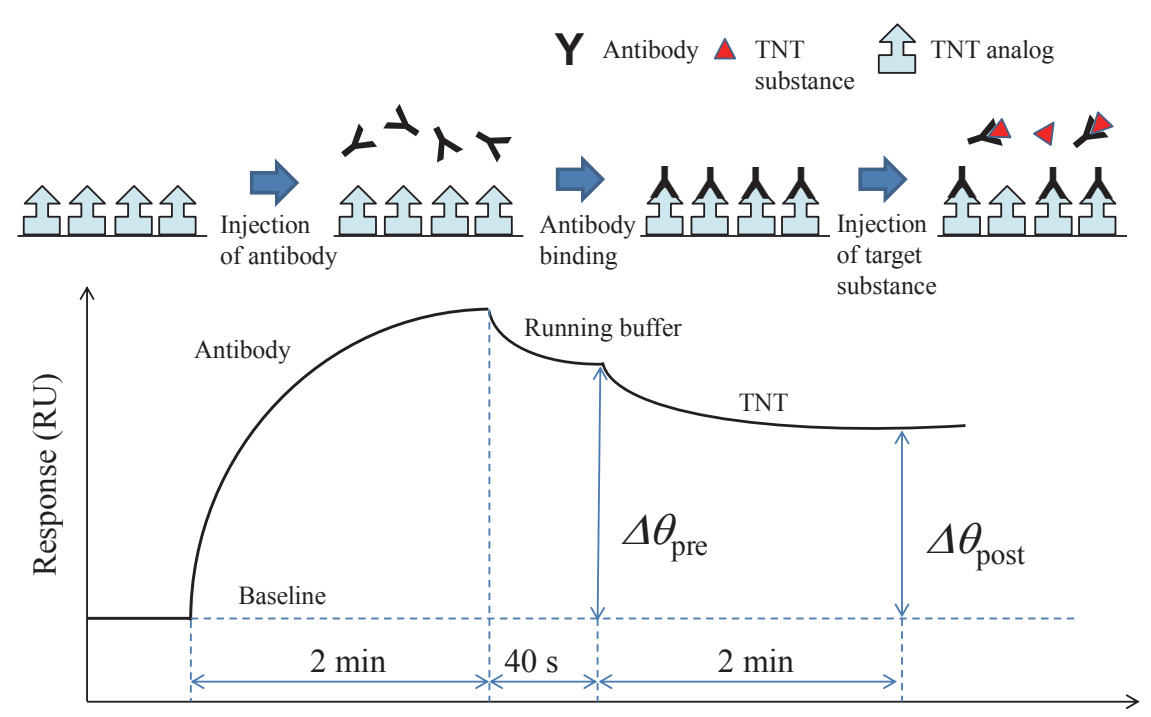

Time (s)

Fig. 3. (Color online) Principle of displacement immunoassay. 
Forty seconds after the end of flowing anti-TNT Ab, PBST (0, reference) and $100 \mathrm{pg} / \mathrm{mL}$ (ppt) and 1 , $5,10,25$, and $50 \mathrm{ng} / \mathrm{mL}$ (ppb) TNT solutions were allowed to flow in this order for $2 \mathrm{~min}$ at $14 \mu \mathrm{L} /$ min. This sequence was performed three times. A $100 \mathrm{ng} / \mathrm{mL}$ (ppb) TNT solution was injected once in the final cycle for the DNP-gly-modified chip. For the 3-NP-aa- and 4-NP-aa-modified chips, 50 and $100 \mathrm{ng} / \mathrm{mL}$ were measured at once.

\section{Results and Discussion}

\subsection{Evaluation of synthesized polymer}

Synthesized polymers were evaluated by ATR-FTIR spectroscopy. The results are shown in Fig. 4. The poly-NVF result shows an adsorption peak from 1570 to $1515 \mathrm{~cm}^{-1}$ due to $\mathrm{N}-\mathrm{H}$ bending vibrations of the amide bond and two peaks at around $1400 \mathrm{~cm}^{-1}$ due to $\mathrm{C}-\mathrm{N}$ stretching vibrations. ${ }^{(10)}$ In contrast, the peaks were decreased for poly-VAm, because the polymer was hydrolyzed with $2 \mathrm{M} \mathrm{NaOH}$ for $4 \mathrm{~h}$. Therefore, hydrolysis was complete. The extent of hydrolysis of poly-VAm was defined as $100 \%$. As a result of the polymer being hydrolyzed with $2 \mathrm{M} \mathrm{NaOH}$ for 10 min, the peaks considerably decreased. The peak at $1525 \mathrm{~cm}^{-1}$ decreased to about $40 \%$ of the polyNVF peak. The polymer has not only vinylformamide but also an amine group. The polymer is a copolymer, poly-(NVF-co-VAm). Therefore, the extent of hydrolysis of the polymer was defined as $60 \%$. Succinic anhydride reacted with amine groups of poly-(NVF-co-VAm) in a ring-opening process, creating an amide bond and forming a terminal carboxyl group. ${ }^{(11)}$ The $60 \%$ hydrolyzed polymer that reacted with succinic anhydride showed increasing adsorption peaks from the level of $60 \%$ hydrolyzed polymer without succinic acid. Thus, it is likely that amide bonds were formed between the amine groups of poly-(NVF-co-VAm) and the succinic acid.

\subsection{Evaluation of nonspecific adsorption of a sensor chip modified with the polymer}

The results of nonspecific adsorption for a sensor chip modified with the poly-(NVF-co-VAm) $(60 \%$ hydrolyzed poly-NVF) are shown in Fig. 5. A resonance unit (RU) of 2800 for the binding amount for anti-TNT Ab was observed. In our previous study, a sensor chip modified with 50-60\%

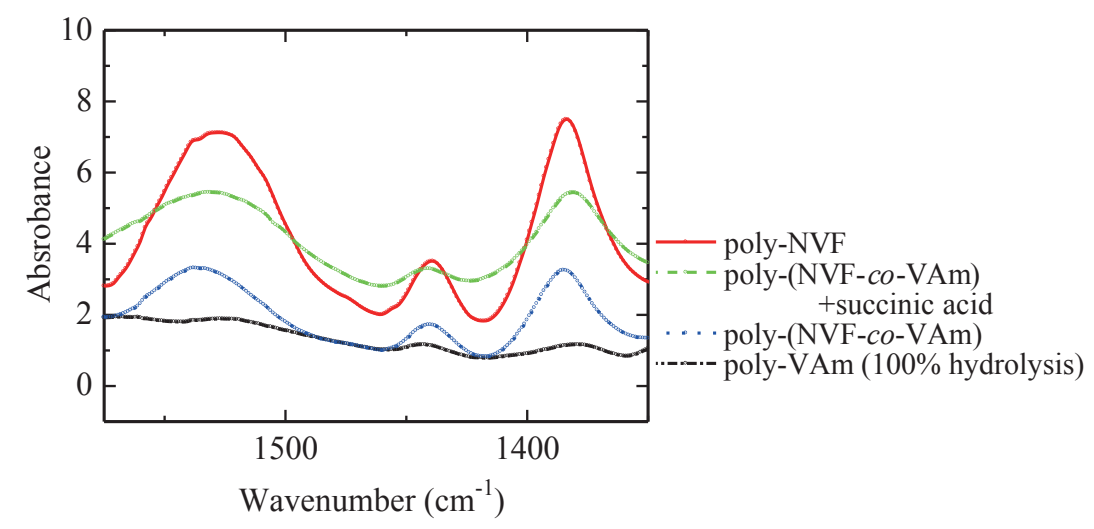

Fig. 4. (Color online) ATR-FTIR spectra of four types of synthesized polymer. 
hydrolysis of poly-(NVF-co-VAm) exhibited a binding amount of $16000 \mathrm{RU}$ to anti-TNT Ab. ${ }^{(5)}$ An RU of 2800 for anti-TNT Ab solution was bound to DNP-gly immobilized on the polymer surface. The reduction of binding amounts of the antibody might be due to steric hindrance owing to the density of the immobilized polymer. The degree of polymerization of poly-NVF could be different. The adsorption of lysozyme to the surface was very low. However, the adsorption of 150 $\mathrm{RU}$ was observed for BSA. BSA is negatively charged at neutral $\mathrm{pH}$ and was probably bound to the positively charged amine group of the polymer. Almost no sensor response for lysozyme was observed.

The results of nonspecific adsorption for a sensor chip modified with the poly-(NVF-co-VAm) with succinic acid aere shown in Fig. 6. The adsorption amounts of high concentrations of BSA and lysozyme were both about $30 \mathrm{RU}$. The adsorption of BSA was reduced to one-fifth of the result on the sensor chip modified with the $60 \%$ hydrolyzed poly-NVF. It was possible that the amine group was converted to an amide bond owing to succinic anhydride, and then the sensor chip surface was almost electrically neutralized. More than $5000 \mathrm{RU}$ of a binding amount of anti-TNT Ab was obtained using the sensor chip. Thus, we chose the sensor chip modified with poly-(NVF-co-VAm) with succinic acid.

\subsection{Detection of TNT}

We chose DNP-gly, 3-NP-aa, and 4-NP-aa as TNT analogs for anti-TNT Ab binding sites. Affinity to dinitroaromatic compounds of anti-TNT Ab used in this study was lower than that to trinitroaromatic compounds. ${ }^{(12)}$ We expected to obtain a higher sensitivity to TNT by displacement immunoassay using a weaker interaction of anti-TNT Ab to mononitro compounds. Figure 7 shows overlaid sensorgrams recorded using the DNP-gly-modified sensor surface. The vertical axis indicates the sensor response in RU. A resonance angle shift of $0.1^{\circ}$ was defined as $1000 \mathrm{RU}$. . $^{(13)}$ After injecting a $25 \mu \mathrm{g} / \mathrm{mL}$ ( $25 \mathrm{ppm}$ ) antibody solution, the sensor response increased rapidly with the binding of the antibody to the sensor surface for $2 \mathrm{~min}$. The sensor response reached about $5000 \mathrm{RU}$. After the antibody flow, the sensor response slightly decreased owing to spontaneous

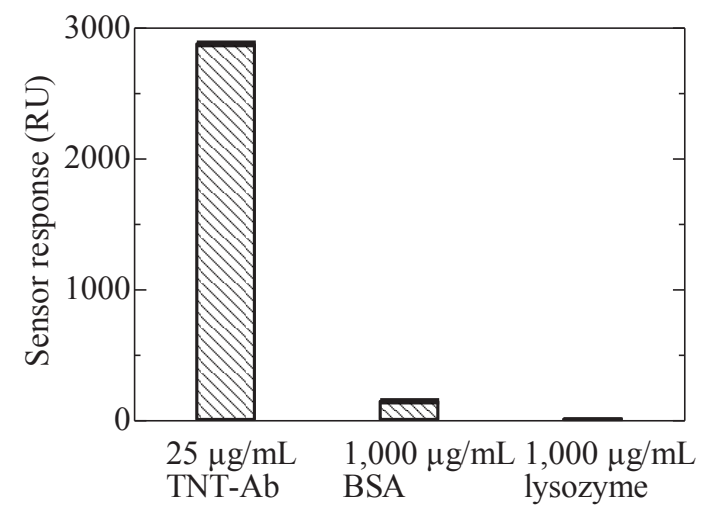

Fig. 5. Binding amount of anti-TNT $\mathrm{Ab}$ and adsorption amounts of BSA and lysozyme on the poly-(NVF-co-VAm).

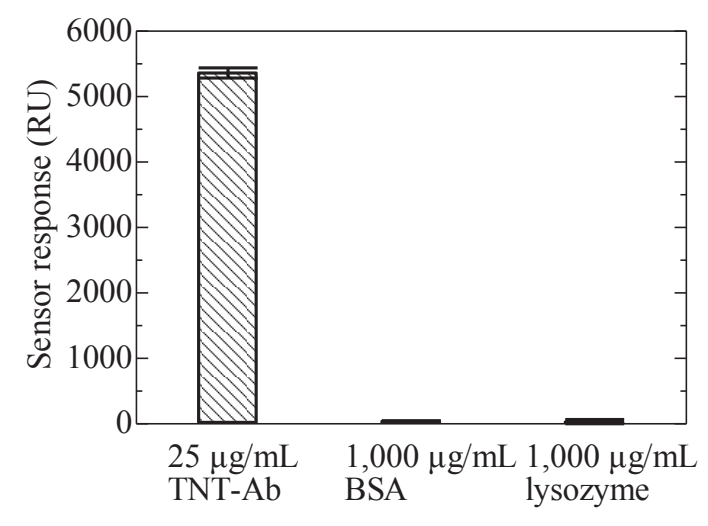

Fig. 6. Binding amount of anti-TNT $\mathrm{Ab}$ and adsorption amounts of BSA and lysozyme on the poly-(NVF-co-VAm) reacted with succinic acid. 


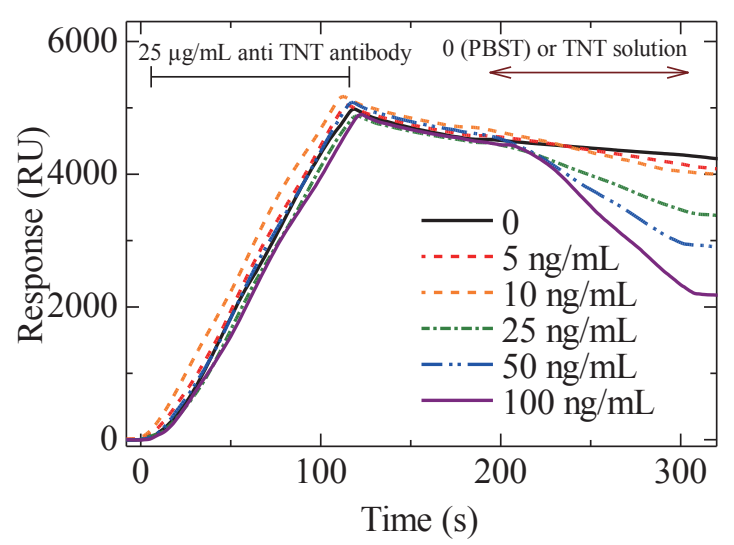

Fig. 7. (Color online) Overlaid sensorgrams of displacement immunoassay using $25 \mu \mathrm{g} / \mathrm{mL}$ anti-TNT $\mathrm{Ab}$ to detect varying concentrations of TNT on DNPgly-modified sensor chip.

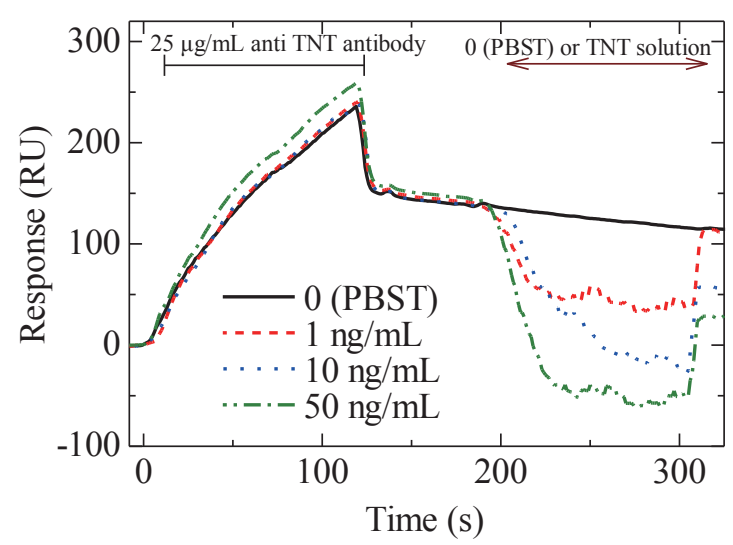

Fig. 8. (Color online) Overlaid sensorgrams of displacement immunoassay using $25 \mu \mathrm{g} / \mathrm{mL}$ anti-TNT $\mathrm{Ab}$ to detect varying concentrations of TNT on 3-NPacetic acid-modified sensor chip.

dissociation in $40 \mathrm{~s}$. A TNT injection at greater than or equal to $25 \mathrm{ng} / \mathrm{mL}$ caused a large decrease in response.

SDS $(0.5 \%)$ was suitable for the regeneration of the sensor surface. When $50 \mathrm{mM} \mathrm{NaOH}$ was used as a regeneration solution, hydrolysis of the modified polymer proceeded with each regeneration cycle, after which the stability of the binding amount became worse.

Figure 8 shows overlaid sensorgrams using the 3-NP-aa-modified sensor surface. Response curves for TNT flows of 0 and $1 \mathrm{ng} / \mathrm{mL}$ almost overlap. However, the response curves for 10 and $50 \mathrm{ng} / \mathrm{mL}$ (ppb) TNT flows are clearly different from the 0 and $1 \mathrm{ng} / \mathrm{mL}$ response curves at the end of the flow.

\subsection{Limit of detection of TNT}

Figure 9 shows the displacement immunoassay response curves calculated using Eqs. (1) and (2) obtained on the DNP-gly-, 3-NP-aa-, and 4-NP-aa-modified surfaces. In the range of 10-50 ng/mL (ppb) TNT, the 3-NP-aa-modified surface displacement ratio was higher than the DNP-gly-modified surface displacement ratio. The standard deviation (SD) was very low for these sensor chips. However, the standard deviations were large on the 4-NP-aa-modified sensor chip.

The amounts of bound antibody at $25 \mu \mathrm{g} / \mathrm{mL}$ and the determined LODs for TNT on each sensor chip are summarized in Table 1. Although 4696 RU of anti-TNT Ab can be bound to the DNPgly-modified chip at $25 \mu \mathrm{g} / \mathrm{mL}$, only 145 and $55 \mathrm{RU}$ were obtained on the 3-NP-aa- and 4-NP-aamodified chips, respectively. In our previous study, $180 \mathrm{RU}$ was obtained with binding $25 \mu \mathrm{g} / \mathrm{mL}$ anti-TNT Ab to a dinitrophenyl acetic acid-modified chip. That binding amount was lower than that of a DNP-gly-modified chip. The ease of immobilization to a polymer modified on sensor chips may relate to the structures of these TNT analogs.

We did not determine the LOD on the 4-NP-aa-modified chip because the SD was very large. The binding amount of anti-TNT Ab was not stable for each measurement cycle. The LODs of TNT on the DNP-gly- and 3-NP-aa-modified chips were obtained as 2.8 and $0.9 \mathrm{ppb}$, respectively. 


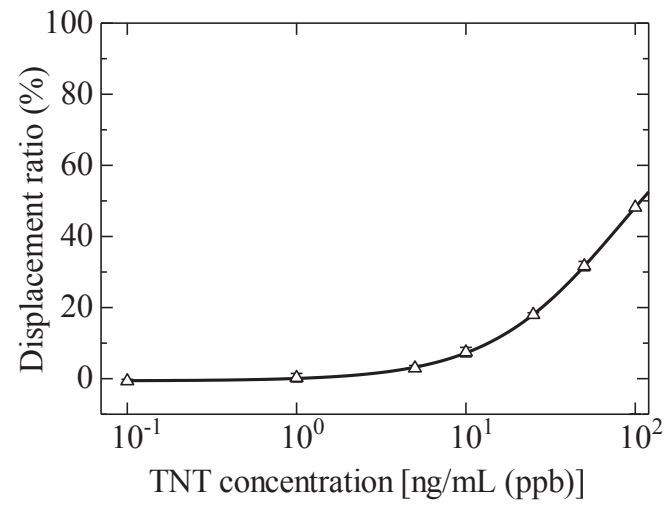

(a)

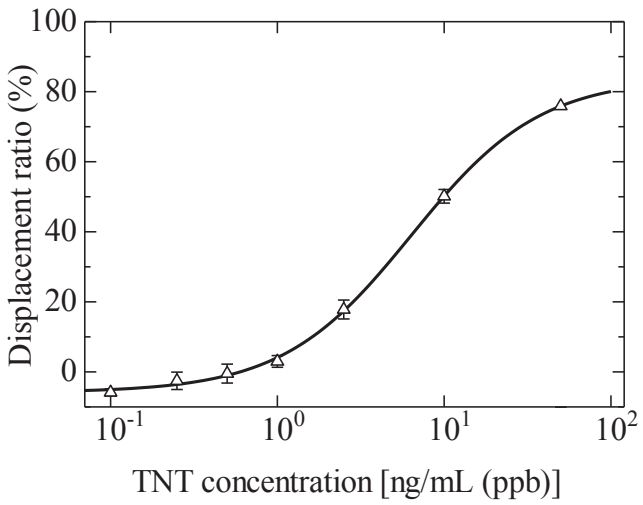

(b)

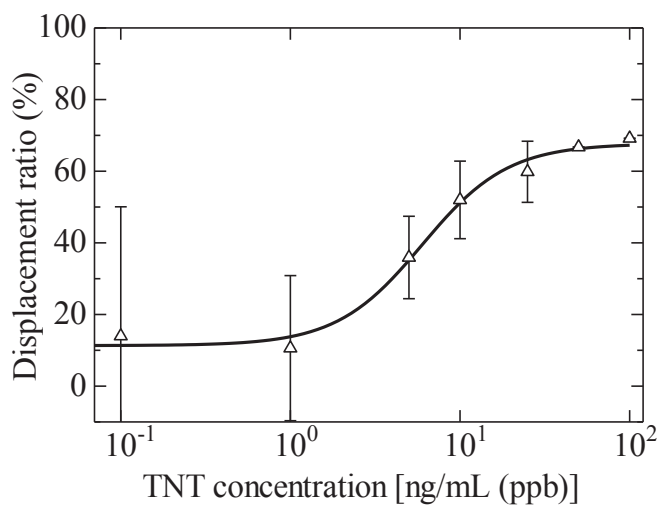

(c)

Fig. 9. Response curves obtained by displacement assay of three types of TNT analog-immobilized sensor surfaces. (a) DNP-gly-modified sensor chip, (b) 3-NP-aa-modified sensor chip, and (c) 4-NP-aa-modified sensor chip.

Table 1

Binding amounts of $25 \mu \mathrm{g} / \mathrm{mL}$ anti-TNT Ab and LODs were considered at 3 SDs for each TNT analog sensor chip.

\begin{tabular}{lccc}
\hline & \multicolumn{3}{c}{ TNT analog } \\
\cline { 2 - 4 } & DNP-gly & 3-NP-aa & 4-NP-aa \\
\hline Bound antibody to 25 ppm antibody (RU) & 4696 & 145.5 & 54.8 \\
Limit of detection (ng/mL or ppb) & 2.8 & 0.9 & - \\
\hline
\end{tabular}

\section{Conclusions}

We developed a sensor surface modified with a polymer, poly-(NVF-co-VAm) reacted with succinic anhydride, for the detection of TNT by displacement immunoassay on an SPR sensor system. DNP-gly modified via the polymer on a sensor chip suppressed the nonspecific adsorption of BSA and lysozyme, and then anti-TNT Ab could be bound to the sensor chip selectively. The limit of TNT detection was investigated for three immobilized TNT analogs, namely DNP-gly, 3-NP-aa, and 4-NP-aa. The 3-NP-aa-modified sensor surface showed the lowest LOD among 
these three sensor chips. A LOD of $0.9 \mathrm{ppb}$ was achieved. We demonstrated the efficacy of a mononitroaromatic compound utilized as an anti-TNT Ab binding site for the detection of TNT. LOD and sensitivity will be improved by tuning the sensor surface conditions in the near future.

\section{Acknowledgements}

This work was partly supported by a Grant-in-Aid for Scientific Research (C)(26420310) from the Japan Society for the Promotion of Science (JSPS).

\section{References}

1 T. Onodera: Electronic Dog Nose Using Surface Plasmon Resonance Method: In Biochemical Sensors: Mimicking Gustatory and Olfactory Senses (Pan Stanford Publishing, Singapore, 2013) p. 351.

2 T. Onodera and K. Toko: Sensors 14 (2014) 16586.

3 T. Onodera, Y. Mizuta, K. Horikawa, P. Singh, K. Matsumoto, N. Miura, and K. Toko: Sens. Mater. 23 (2011) 39.

4 R. Yatabe, T. Onodera, and K. Toko: Front Bioeng Biotechnol 2 (2014) 10.

5 R. Yatabe, T. Onodera, and K. Toko: Sens. Mater. 25 (2013) 45.

6 M. Yasuura, K. Toko, and T. Onodera: Sens. Mater. 23 (2011) 21.

7 K. Yamamoto, Y. Imamura, E. Nagatomo, T. Serizawa, Y. Muraoka, and M. Akashi: J. Appl. Polym. Sci. 89 (2003) 1277.

8 H. Kitano, T. Kondo, T. Kamada, S. Iwanaga, M. Nakamura, and K. Ohno: Colloids Surf. B. Biointerfaces 88 (2011) 455.

9 S. Y. Rabbany, W. J. Lane, W. A. Marganski, A. W. Kusterbeck, and F. S. Ligler: J. Immunol. Methods 246 (2000) 69.

10 S. Araki, Y. Mashiko, O. Yamamoto, and T. Kamada (translated): Spectrometric Identification of Organic Compounds (Tokyokagakudojin, Tokyo, Japan, 2006) 7th ed. p. 107.

11 G. T. Hermanson: Bioconjugate Techniques (Elsevier Academic Press, MA, USA, 2008) 2nd ed. p. 102.

12 A. Zeck, M. G. Weller, and R. Niessner: Fresenius J. Anal. Chem. 364 (1999) 113.

13 E. Stenberg, B. Persson, H. Roos, and C. Urbaniczky: J. Colloid Interface Sci. 143 (1991) 513. 